\title{
Adolescent Patients Exhibit Significant Improvements in Strength and Functional Performance From 6 to 9 Months After ACL Reconstruction With Quadriceps Autograft
}

\author{
Michael Saper, D.O., A.T.C., C.S.C.S., Chris Wong, P.T., D.P.T., S.C.S., C.S.C.S., and \\ Nicole Strauss, M.P.H.
}

Purpose: To investigate differences between the 6-month and 9-month return to sport (RTS) assessments in adolescent patients undergoing anterior cruciate ligament reconstruction (ACLR) with quadriceps tendon autograft. Methods: A retrospective review was performed of consecutive adolescent patients who underwent ACLR between June 2017 and October 2019. Patients completed a structured RTS test 6 and 9 months after surgery consisting of isometric and isokinetic strength testing, the Lower Quarter Y-Balance Test, and single-legged hop testing. The recovery of muscle strength, assessed via isometric/isokinetic and hop testing, was defined by a limb symmetry index (LSI) $\geq 90 \%$. Differences were compared between the 2 RTS test time points. Results: In total, 27 patients ( 16 female, 11 male) were identified with a mean age of 15 years (range, 13-17 years). There were statistically significant improvements in isometric quadriceps strength (LSI, $85.0 \pm 17.2$ vs $92.5 \pm 11.3 ; P=.04)$ and hamstring strength (LSI, $87.9 \pm 11.2$ vs $99.0 \pm 10.5 ; P<.01)$. There were improvements in isokinetic knee extension at both $60^{\circ} / \mathrm{s}$ (LSI, $75.2 \pm 16.7 \mathrm{vs} 83.3 \pm 13.8$ ) and $180^{\circ} / \mathrm{s}$ (LSI, $79.9 \pm$ 15.4 vs $83.4 \pm 11.2)$, but the differences were only statistically significant at $60^{\circ} / \mathrm{s}(P=.02$ and $P=.17$, respectively). There were no significant differences in isokinetic testing of knee flexion at either $60^{\circ} / \mathrm{s}$ or $180^{\circ} / \mathrm{s}$. There were no statistically significant differences in the anterior reach component of the Lower Quarter Y-Balance Test at 6 and 9 months. Patients demonstrated statistically significant improvements on single- legged hop testing with mean LSIs $>95 \%$ for each of the 4 tests at 9 months postop. Conclusions: Adolescent patients undergoing ACLR with quadriceps tendon autograft demonstrated significant improvements in quadriceps strength between 6 and 9 months postoperatively. Level of Evidence: Level IV, therapeutic case series.

A nterior cruciate ligament (ACL) tears are most common among adolescent patients (13-20 years old) compared with adults older than 20 years old. ${ }^{1,2}$

From the Departments of Orthopedics and Sports Medicine (M.S., N.S.) and Rehabilitation Medicine (C.W.), Seattle Children's; and Department of Orthopaedics and Sports Medicine, University of Washington (M.S.), Seattle, Washington, USA.

The authors report the following potential conflicts of interest or sources of funding: M.S. receives research support from Arthrex, outside the submitted work. Full ICMJE author disclosure forms are available for this article online, as supplementary material.

Received August 8, 2020; accepted January 27, 2021.

Address correspondence to Michael G. Saper, D.O., A.T.C., C.S.C.S., 4800 Sand Point Way NE, Seattle, WA 98105.E-mail: Michael.Saper@ seattlechildrens.org

(C) 2021 THE AUTHORS. Published by Elsevier Inc. on behalf of the Arthroscopy Association of North America. This is an open access article under the CC BY-NC-ND license (http://creativecommons.org/licenses/by-nc-nd/4.0/). 2666-061X/201357

https://doi.org/10.1016/j.asmr.2021.01.026
With increasing primary anterior cruciate ligament reconstructions (ACLRs) comes a rise in revision surgery, particularly in the adolescent population, which also has been shown to be at a greater risk for graft rupture than adults. ${ }^{2,3}$ Failure rates in this young population ( $25 \%$ or more in some reports) could be due to a myriad of factors, including body mass index, sport and competition level, graft type, and time to return to sport (RTS) ${ }^{2,3}$ Furthermore, return to preinjury level of sport this high-risk population is around $80 \%{ }^{4}$

Postoperative rehabilitation is vital for a successful outcome after ACLR. ${ }^{5,6}$ Optimal rehabilitation consists of both time and goal-based protocols that apply appropriate levels of stress to healing tissues, address postoperative impairments, include sport-specific training, and assess readiness to RTS. ${ }^{5,7}$ For surgeons and physical therapists, readiness to RTS and the risk of reinjury often is assessed with objective measurements from RTS tests examining limb symmetry and muscle 
strength and dynamic functional activity. ${ }^{5,8}$ To minimize reinjury risk, some have advocated delaying RTS to at least 9 months after ACLR. ${ }^{9,10}$ Beischer et al. ${ }^{9}$ found that RTS before 9 months after ACLR was associated with an approximately 7 -fold increased rate of sustaining a second ACL injury. Grindem et al. ${ }^{7}$ reported that delaying RTS from 6 to 9 months after surgery decreased the risk of reinjury by $51 \%$ each month RTS was delayed. Yet, previous authors have shown that the percentages of patients passing all RTS criteria were low at both 6 and 9 months after ACLR. ${ }^{4,11,12}$ In addition, they found a lack of improvement in quadriceps strength and patientreported outcomes (PROs) 9 months following surgery.

Recently, ACLR using quadriceps tendon autograft (QT) has gained in popularity. ${ }^{13,14}$ Advantages include similar functional outcomes and lower failure rates compared with other soft-tissue grafts. ${ }^{13}$ However, studies are lacking that evaluate RTS testing after ACLR with QT. The purpose of this study was to investigate differences between the 6-month and 9-month RTS assessments in adolescent patients undergoing ACLR with QT. It was hypothesized that significant improvements in strength and functional performance would be observed in this young population from 6 to 9 months after ACLR, but that limb asymmetries would persist up to 9 months following surgery.

\section{Methods}

\section{Patient Selection}

Institutional review board approval was obtained before initiation of this study. A retrospective review was performed of a group of consecutive adolescent patients who underwent ACLR between June 2017 and October 2019. All surgeries were performed by a single pediatric and sports medicine fellowship-trained orthopaedic surgeon (M.S.). Patients were included if they (1) underwent primary ACLR with all soft-tissue QT using an all-inside technique ${ }^{15}$ and (2) had formal postoperative RTS assessments at both 6 and 9 months following surgery. Patients with meniscus repairs, partial meniscectomies, and articular cartilage debridements were included. Exclusion criteria for this study were (1) patients with concomitant ligament injuries requiring repair/reconstruction (e.g., lateral extra-articular tenodesis), and (2) those with a history of previous ipsilateral or contralateral lower extremity surgery.

\section{Postoperative Protocol}

All patients followed the same standardized postoperative rehabilitation protocol. ${ }^{15}$ The postoperative protocol was unchanged in the setting of concomitant meniscus repair. Weight-bearing as tolerated was allowed immediately postoperatively in a hinged-brace locked in full extension. The brace was worn during the first 6 weeks. It was kept locked in extension for the first 2-4 weeks. Formal postoperative rehabilitation was initiated 3-4 days following surgery and consisted of 6 separate phases (Appendix Table 1, available at www. arthroscopyjournal.org). During the first week, rangeof-motion (ROM) exercises were initiated and exercises to re-establish quadriceps and hamstring control and activation via isometric contractions commenced. During Phase 2, patients worked to gradually improve ROM (ideally $90^{\circ}$ by week 4) with the incorporation of closed kinetic lower-extremity quadriceps and gluteus exercises. Hamstring concentric and eccentric activation exercises without resistance also were employed in this Phase 2. Phase 3 goals included working to gradually restore full ROM; restoring muscular strength with progressive, resistive hamstring strengthening and advanced closed kinetic chain resistive exercises; enhancing neuromuscular control. Phases 4 and 5 focused on strength, power, and endurance, as well as the progression of functional activities. Flat-ground running was not started before 4 months postoperative, but pre-running and light impact activities focusing on biomechanics were initiated at 10-12 weeks.

\section{RTS Assessments}

RTS assessments were routinely performed at both 6 and 9 months postoperatively at one of our institution's sports rehabilitation clinics by a licensed physical therapist. Isometric strength was assessed using a handheld digital dynamometer (MicroFET2, Salt Lake City, UT). ${ }^{16}$ Peak torques for knee extension and flexion were collected with the patient sitting and the knee at $90^{\circ}$ of flexion. Peak torque for hip abduction was collected with the patient in the lateral decubitus position and the hip at $30^{\circ}$ of abduction. Isokinetic strength was assessed using a Biodex System 3 (Biodex Medical Systems, Shirley, NY) computerized robotic dynamometer. Peak torques for knee extension and flexion were evaluated at angular speeds of 60 and $180^{\circ} / \mathrm{s}$. All isometric and isokinetic strength assessments were performed bilaterally. Lower-extremity strength, neuromuscular control, flexibility, and balance were assessed with the Lower Quarter Y-Balance Test (YBTLQ). Assessment of function and dynamic strength/ stability was performed using 4 different single-leg hop tests: single hop for distance, triple hop for distance, triple crossover hop for distance, and timed hop.

Limb symmetry indices (LSIs) were calculated for all strength and hop tests using the following: LSI = (surgical/uninvolved) $\times 100 \%$. Recovery of muscle strength was defined by a LSI $\geq 90 \%$. HS: Q ratios $>50 \%$ were considered passing. For the YBT-LQ, a side-to-side difference of $<4 \mathrm{~cm}$ on the anterior reach and a composite score $>90$ on each limb were criteria 
Table 1. Demographics

\begin{tabular}{lc}
\hline \multicolumn{1}{c}{ Characteristic } & Data \\
\hline Age at surgery, y & $15(13-17)$ \\
Sex & \\
Female & $16(59.3)$ \\
Male & $11(40.7)$ \\
Height, cm & $168.1 \pm 8.2$ \\
Weight, kg & $66.9 \pm 10.9$ \\
BMI & $23.5 \pm 3.6$ \\
Primary sport & \\
Soccer & $9(33.3)$ \\
Basketball & $6(22.2)$ \\
Football & $5(18.5)$ \\
Skiing & $2(7.4)$ \\
Volleyball & $2(7.4)$ \\
Softball & $1(3.7)$ \\
Tennis & $1(3.7)$ \\
Ultimate frisbee & $1(3.7)$ \\
Preoperative patient-reported outcomes & \\
Pedi-IKDC (n = 26) & $56.5 \pm 11.8$ \\
Lysholm (n = 25) & $72.0 \pm 16.4$ \\
Tegner (n = 25) & $2.1 \pm 1.1$ \\
\hline NOTE. Data are reported as meanternational Knee \\
(range), or n (\%) \\
BMI, body mass index; Pedi-IKDC, Pediatric \\
Documentation Committee. \\
$\quad$ \\
\hline
\end{tabular}

for passing. Passing the dynamic testing component of the RTS assessment was defined as LSIs $\geq 90 \%$ on all 4 components of the hop tests. ${ }^{11,12}$

Medical records were reviewed and demographic data (e.g., age at surgery, height, and body mass index) were collected. Details of the surgical intervention (e.g., concomitant procedures), and postoperative follow-up information were recorded. PROs were evaluated using the Pediatric International Knee Documentation Committee (Pedi-IKDC) Subjective Knee Evaluation Form, ${ }^{17}$ Lysholm Knee Questionnaire, ${ }^{18}$ and the Tegner Activity Scale. ${ }^{18}$ Data from the RTS tests were tabulated from the physical therapy notes.

\section{Statistical Analysis}

Descriptive statistics were calculated. Continuous variables (i.e., age) were reported as means \pm standard deviations. Categorical variables (i.e., sex) were reported as frequencies with percentages. All data were tabulated in Excel (version 2012; Microsoft, Redmond, WA). Continuous dependent variables were checked for normality of distribution using the Shapiro-Wilk test. A power analysis with an $\alpha$ of 0.05 and power of 0.8 found that a minimum sample size of 27 was required to detect statistical significance. As the data were normally distributed, differences were compared between the 2 RTS time points using paired t-tests. $\chi^{2}$ tests were used to compare the proportion of patients passing each return to sport criterion. Statistical significance was set at $P<.05$. All analyses were performed using STATA 14.2 (StataCorp, College Station, TX).

\section{Results}

Fifty-eight patients underwent primary ACLR with QT during the study period; 9 were lost to follow-up. Two patients were excluded (one with a concomitant medial collateral ligament repair and the other with a lateral extra-articular tenodesis). A total of 27 patients (16 female, 11 male) had complete RTS test data and were ultimately included with a mean age of 15 years (range, 13-17 years). The demographic characteristics are summarized in Table 1. In total, $14.8 \%$ of patients underwent isolated ACLR. Concomitant all-inside medial or lateral meniscus repairs were performed in $33.3 \%$ and $44.4 \%$ of patients, respectively.

The length of time from surgery to the respective RTS assessments was $6.0 \pm 0.3$ months and $9.1 \pm 0.5$ months. From 6 to 9 months postoperatively, there were statistically significant improvements in the PediIKDC $(84.3 \pm 8.5$ vs $93.6 \pm 7.3 ; P<.01)$ and Lysholm scores $(93.7 \pm 6.6$ vs $98.2 \pm 3.0 ; P<.01)$. The Tegner activity scale also improved from 6 to 9 months $(5.7 \pm 2$ vs $7.0 \pm 2.1 ; P<.01)$ postoperatively.

There were statistically significant improvements in isometric quadriceps strength (LSI, $85.0 \pm 17.2$ vs $92.5 \pm 11.3 ; P=.04$ ) and hamstring strength (LSI, $87.9 \pm 11.2$ vs $99.0 \pm 10.5 ; P<0.01$ ) (Table 1). There were improvements in isokinetic knee extension at both $60^{\circ} / \mathrm{s}$ (LSI, $75.2 \pm 16.7$ vs $83.3 \pm 13.8$ ) and $180^{\circ} / \mathrm{s}$ (LSI, $79.9 \pm 15.4$ vs $83.4 \pm 11.2$ ), but the differences were only statistically significant at $60^{\circ} / \mathrm{s}(P=.02$ and

Table 2. Isometric and Isokinetic Strength Testing LSIs

\begin{tabular}{lccc}
\hline & 6 Months & 9 Months & $P$ Value \\
\hline Isometric knee extension & $85.0 \pm 17.2$ & $92.5 \pm 11.3$ & $\mathbf{. 0 4}$ \\
Isometric knee flexion & $87.9 \pm 11.2$ & $99.0 \pm 10.5$ & $\mathbf{0 1}$ \\
Isometric hip abduction & $101.7 \pm 13.3$ & $104.3 \pm 7.3$ & .40 \\
Isokinetic knee extension $\left(60^{\circ} / \mathrm{s}\right)$ & $75.2 \pm 16.7$ & $83.3 \pm 13.8$ & $\mathbf{. 0 2}$ \\
Isokinetic knee flexion $\left(60^{\circ} / \mathrm{s}\right)$ & $104.0 \pm 21.7$ & $83.0 \pm 23.2$ & .85 \\
Isokinetic knee extension $\left(180^{\circ} / \mathrm{s}\right)$ & $79.9 \pm 15.4$ & $96.8 \pm 11.2$ & .17 .9 \\
Isokinetic knee flexion $\left(180^{\circ} / \mathrm{s}\right)$ & $95.3 \pm 18.2$ & .75 \\
\hline
\end{tabular}

NOTE. Data reported as mean \pm standard deviation. Bolded $P$ value indicates statistical significance.

LSI, limb symmetry index. 
Table 3. Isometric and Isokinetic HS:Q Ratios

\begin{tabular}{lccc}
\hline & 6 Months & \multicolumn{1}{c}{9 Months } & $P$ Value \\
\hline Isometric (surgical) & $63.8 \pm 11.4$ & $61.8 \pm 12.1$ & .61 \\
Isometric (uninvolved) & $63.1 \pm 21.0$ & $61.5 \pm 14.3$ & .75 \\
Isokinetic $60^{\circ} / \mathrm{s}$ (surgical) & $71.6 \pm 15.4$ & $62.7 \pm 11.4$ & .07 \\
Isokinetic $60^{\circ} / \mathrm{s}$ (uninvolved) & $51.3 \pm 10.5$ & $50.7 \pm 9.4$ & .84 \\
Isokinetic $180^{\circ} / \mathrm{s}$ (surgical) & $66.1 \pm 17.1$ & $67.6 \pm 11.3$ & .74 \\
Isokinetic $180^{\circ} / \mathrm{s}$ (uninvolved) & $53.9 \pm 10.0$ & $55.4 \pm 11.8$ & .62
\end{tabular}

NOTE. Data reported as mean \pm standard deviation. Bolded $P$ value indicates statistical significance.

HS:Q, hamstrings/quadriceps ratio.

$P=.17$, respectively) (Table 2). Isometric and isokinetic hamstrings/quadriceps (HS:Q) ratios are presented in Table 3. There was a statistically significant difference in the anterior reach component of the YBT-LQ at 6 and 9 months $(P<.01)$ (Table 4$)$. Patients demonstrated statistically significant improvements on single-legged hop testing with mean LSIs $>95 \%$ for each of the 4 tests at 9 months postop (Table 5). Table 6 presents the percentages of patients who passed specific RTS criteria at the 6- and 9-month assessments. A statistically significant greater proportion of patients passed all 4 hop tests at 9 months compared with 6 months $(P<.01)$. In addition, more patients passed the YBT-LQ anterior reach $(P<001)$ and isometric knee flexion $(P<001)$ at 9 months compared with 6 months. No patients passed all 20 criteria at either time point.

The mean time to RTS clearance was $9.5 \pm 1.0$ months. Twenty patients $(74.1 \%)$ were given clearance to progress RTS activities after their 9-month assessment. Three patients repeated the strength and functional assessment at 12 months postoperatively and were subsequently cleared to RTS. Four patients have yet to repeat a subsequent test for clearance for RTS. The mean follow-up after surgery was $16.4 \pm 7.8$ months (range, 9-36 months). At the 9-month RTS assessment, all patients had a physical examination to assess knee stability. All patients had normal Lachman examinations. In total, 24 patients $(88.9 \%)$ had negative pivot shifts; 3 patients $(11.1 \%)$ had grade 1 pivot shifts. There was no difference in knee stability between the 6- and 9-month assessments. One patient ruptured his graft 3 years postoperatively following a skiing accident. Another patient sustained a contralateral ACL injury 25.5 months after surgery due to a contact injury playing soccer. A third patient sustained an ipsilateral patella subluxation 36 months postoperatively and was managed conservatively.

\section{Discussion}

The principal findings of this study were that there were improvements in strength and functional performance from 6 to 9 months after ACLR with QT. There were statistically significant increases in the percentages of patients who passed key RTS parameters (isometric quadriceps and hamstring strength, anterior reach component of the YBT-LQ, and single-legged hop testing). However, limb asymmetries persisted up to 9 months postoperatively after ACLR, particularly with isokinetic quadriceps strength at 60 and $180^{\circ}$ s. These findings suggest that surgeons, physical therapists, and athletic trainers should exercise caution with relying solely on time-based criteria for progression through ACLR rehabilitation and ultimately RTS decisionmaking. Rehabilitation should progress at a rate specific for each patient and strength and functional testing should be used to help determine readiness for RTS. In the adolescent population, strong consideration should be given to delay RTS for at least 9 months after ACLR with QT.

To our knowledge, there are limited data regarding results of strength and functional performance testing after ACLR with QT, especially in adolescent patients. A low HS:Q ratio is a known associated risk factor for secondary ACL injuries. The results of this study were similar to those of Martin-Alguacil et al., ${ }^{19}$ which found greater HS:Q ratios in QT versus hamstring autograft (HS) ACLRs at 3, 6, and 12 months in a group of soccer players. Furthermore, HS:Q ratios in the current study were similarly maintained at $>60 \%$ at 6 and 9 months. Fischer et al. ${ }^{20}$ compared isokinetic $\left(60^{\circ} / \mathrm{s}\right)$ quadriceps and hamstring muscle strength in 141 young patients (mean age, 22 years) after ACLR with either HS or QT. Their results indicated the QT group had statistically significantly greater HS:Q ratios in the QT group within the first year after surgery. That study also found lower quadriceps strength during the first year compared with the HS group. ${ }^{20}$ This may be interpreted as a reason for a greater HS:Q ratio in the QT group. However, the authors also found that hamstring strength was significantly greater at 5.5 and 7.5 months post-surgery in the QT group. Similarly, Lee et al. ${ }^{21}$ compared isokinetic strength testing between QT and HS ACLR and

Table 4. Lower Quarter Y-Balance Test

\begin{tabular}{lrrr}
\hline & 6 Months & \multicolumn{1}{c}{9 Months } & $P$ Value \\
\hline Composite score of surgical limb, \% of leg length & $95.2 \pm 17.7$ & $102.4 \pm 6.2$ & .05 \\
Composite score of uninvolved limb, \% of leg length & $100.1 \pm 6.8$ & $103.5 \pm 6.1$ & $1.6 \pm 3.0$ \\
Anterior reach difference, cm & $4.2 \pm 4.3$ & $<.01$ \\
\hline
\end{tabular}

NOTE. Data reported as mean \pm standard deviation. Bolded $P$ value indicates statistical significance. 
Table 5. LSI of Surgical Limb on Hop Testing

\begin{tabular}{llcc}
\hline & \multicolumn{1}{c}{ 6 Months } & 9 Months & $P$ Value \\
\hline Single hop for distance & $89.5 \pm 12.1$ & $95.8 \pm 5.4$ & $<.01$ \\
Triple hop for distance & $90.8 \pm 8.3$ & $96.4 \pm 3.7$ & $<.01$ \\
Crossover hop for distance & $91.6 \pm 11.8$ & $96.8 \pm 3.5$ & .07 \\
Timed hop & $97.0 \pm 11.1$ & $98.5 \pm 4.8$ & .54
\end{tabular}

NOTE. Data reported as mean \pm standard deviation. Bolded $P$ value indicates statistical significance.

LSI, limb symmetry index.

found superior hamstring strength recovery in the QT group. The use of QT for ACLR does not impact the flexor mechanism, and may allow the initiation of isolated, progressive resistive hamstring strength training sooner in the rehabilitation, whereas postoperative physical therapy protocols for HS ACLR may avoid isolated, progressive resistive training for the first 6 to 8 weeks. The overall hamstring strength maintenance and increased strength gains over time may lower the stress on the maturing ACL graft. Lastly, although quadriceps weakness may be evident when comparing QT to HS ACLRs within the first year, quadriceps recovery after QT ACLR is similar to after bone-patellar tendon-bone (BPTB) autograft ACLR. In a study of 30 patients with an average age of 22 years, Hunnicutt et al. ${ }^{22}$ found no significant difference in isokinetic $\left(60^{\circ} / \mathrm{s}\right.$ and $\left.180^{\circ} / \mathrm{s}\right)$ quadriceps strength between graft types at 8 months. This implies that the use of the QT in ACLR, alternative to the BPTB, would have a similar delay in quadriceps recovery. However,
QT use in ACLR may not have the long-term, qualitative adverse repercussions, including patellar tendinopathy or pain while kneeling directly on the graft site, as seen in patients who undergo BPTB procedures.

There were statistically significant improvements in PROs between 6 and 9 months after surgery. The absolute change in Tegner score between the 2 time points was 1.3, which was greater than the minimal detectable change of 1.0. ${ }^{23}$ The absolute change in score for the Lysholm was 4.5, below the minimal clinically important difference of $10.1 .^{24}$ Thus, while statistically significant, the improvement in the Lysholm score is unlikely to be of clinical importance. For the Pedi-IKDC, the absolute change in score was 9.3. While there is no established minimal clinically important difference for the Pedi-IKDC, the nearly 10-point improvement was statistically significant and indicates sufficient self-reported knee function at 9 months after ACLR with QT. As prior investigations have shown lower PROs in patients who did not RTS after ACLR, ${ }^{25}$ these data support the benefit of prolonged rehabilitation beyond 6 months. The findings in this study are contrast to that of Welling et al., ${ }^{26}$ who examined 6- and 9-month RTS tests for 62 patients (mean age, 24.2 years) after ACLR primarily HS and BPTB autografts. The authors found a lack of clinical improvement on the IKDC between the 2 time points after ACLR. Furthermore, the mean score at 9 months was $81.7 \%$ and only $62.9 \%$ of patients were classified as having self-reported knee function with normal ranges.

Table 6. Return-to-Sport Pass Criteria and Percentage of Patients Who Passed

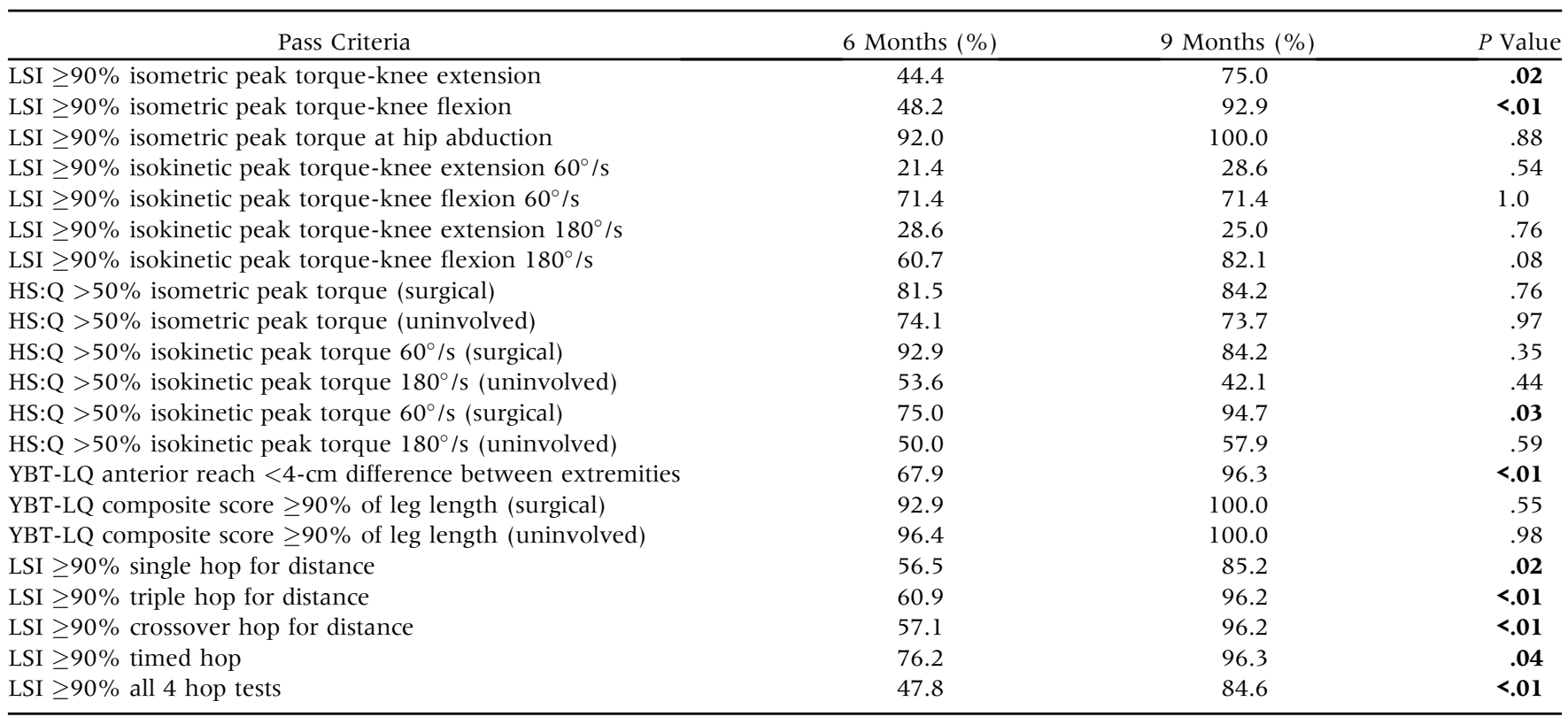

NOTE. Bolded $P$ value indicates statistical significance.

HS:Q, hamstrings/quadriceps ratio; LSI, limb symmetry index, YBT-LQ, Lower Quarter Y-Balance Test. 
Poor dynamic balance is an intrinsic risk factor for lower extremity injury in athletes. The YBT-LQ measures single-limb stance excursion distances while performing lower-extremity dynamic balance testing. Previous work has found that an anterior reach asymmetry greater than $4 \mathrm{~cm}$ may increase lower-extremity injury risk. ${ }^{27}$ At 6 months postoperatively, less than $70 \%$ of patients in this study achieved anterior reach asymmetry $<4 \mathrm{~cm}$ on the YBT-LQ. In contrast, more than $95 \%$ of patients met passing criteria at 9 months postoperatively. This finding indicates that 3 months of additional physical therapy resulted in significant improvements in dynamic balance, potentially lowering the risk of lower-extremity injury upon RTS.

While the mean LSIs on each of the 4 single-leg hop tests met the passing threshold at both 6 and 9 months postoperatively, there was a significant difference in the percentage of patients who passed at 6 versus 9 months. Less than $50 \%$ of patients passed all 4 hop tests at 6 months. Conversely, nearly $85 \%$ of patients passed all 4 hop tests at 9 months postoperatively. These results are similar to those found by Welling et al. ${ }^{24} ; 62.9 \%$ passed all hop tests at 6 months and $77.4 \%$ passed at 9 months. While the ability of hop tests to predict reinjury and posttraumatic knee osteoarthritis has been questioned, they do possess a fair association to PROs and the athlete's ability to RTS after ACLR. ${ }^{28,29}$ The findings in this study emphasize that functional performance after ACLR continues to improve beyond 6 months, and that delaying RTS until 9 months postoperatively will help achieve symmetric muscle function and maximize patients' abilities to RTS.

This study could not (nor was it its purpose) examine the relationship between performance on the RTS tests and reinjury. There is lack of clear evidence for most important re-injury risk factors, and a recent metaanalysis has shown no effect of passing a RTS test battery on overall subsequent ACL injury. ${ }^{12}$ In this study, from 6 to 9 months after ACLR with QT, there were statistically significant increases in the percentages of patients who passed key RTS parameters. Nearly $75 \%$ of patients were cleared to RTS at 9 months, and with an average follow-up of nearly 16.4 months, there was one graft rupture and one contralateral ACL injury.

\section{Limitations}

This retrospective study is not without its limitations. While all patients followed the same rehabilitation protocol, rehabilitation was done at different institutions. It's unclear how such heterogeneity affected the results, and a larger study in the future should take those variables into account. Also, these results reflect the outcomes of patients of a single surgeon at a single children's hospital; a single graft/technique was used, and as such, might not be generalizable to the broader orthopaedic community. Another limitation is that the uninjured limb was used as the control for comparison of the surgically involved limb, which may not truly reflect preinjury baseline strength and function. Normative data or a third control group with asymptomatic knee subjects could be considered in the future. The study's sample size was small resulting in a risk of beta-error with regard to the findings of no significant difference for the secondary outcome measures, and as such, we were unable to analyze the effect of certain variables such as sex or sport. Lastly, this study did not examine correlations between the various test batteries. Such further analyses, while useful, were beyond the scope of this investigation.

\section{Conclusions}

Adolescent patients undergoing ACLR with QT demonstrated significant improvements in quadriceps strength between 6 and 9 months postoperatively.

\section{References}

1. Faunø P, Rahr-Wagner L, Lind M. Risk for revision after anterior cruciate ligament reconstruction is higher among adolescents: Results from the Danish Registry of Knee Ligament Reconstruction. Orthop J Sports Med 2014;2: 2325967114552405.

2. MOON Knee Group, Spindler KP, Huston LJ, Zajichek A, et al. Anterior cruciate ligament reconstruction in high school and college-aged athletes: Does autograft choice influence anterior cruciate ligament revision rates? Am J Sports Med 2020;48:298-309.

3. Wiggins AJ, Grandhi RK, Schneider DK, Stanfield D, Webster KE, Myer GD. Risk of secondary injury in younger athletes after anterior cruciate ligament reconstruction: A systematic review and meta-analysis. Am J Sports Med 2016;44:1861-1876.

4. Kay J, Memon M, Marx RG, Peterson D, Simunovic N, Ayeni OR. Over $90 \%$ of children and adolescents return to sport after anterior cruciate ligament reconstruction: A systematic review and meta-analysis. Knee Surg Sports Traumatol Arthrosc 2018;26:1019-1036.

5. van Melick N, van Cingel REH, Brooijmans F, et al. Evidence-based clinical practice update: Practice guidelines for anterior cruciate ligament rehabilitation based on a systematic review and multidisciplinary consensus. $\mathrm{Br} \mathrm{J}$ Sports Med 2016;50:1506-1515.

6. Kruse LM, Gray B, Wright RW. Rehabilitation after anterior cruciate ligament reconstruction: A systematic review. J Bone Joint Surg Am 2012;94:1737-1748.

7. Grindem H, Snyder-Mackler L, Moksnes H, Engebretsen L, Risberg MA. Simple decision rules can reduce reinjury risk by $84 \%$ after ACL reconstruction: The Delaware-Oslo ACL cohort study. Br J Sports Med 2016;50: 804-808.

8. Werner JL, Burland JP, Mattacola CG, Toonstra J, English RA, Howard JS. Decision to return to sport participation after anterior cruciate ligament reconstruction, part II: Self-reported and functional performance outcomes. J Athl Train 2018;53:464-474. 
9. Beischer S, Gustavsson L, Senorski EH, et al. Young athletes who return to sport before 9 months after anterior cruciate ligament reconstruction have a rate of new injury 7 times that of those who delay return. J Orthop Sports Phys Ther 2020;50:83-90.

10. Kaplan Y, Witvrouw E. When is it safe to return to sport after ACL reconstruction? Reviewing the criteria. Sports Health 2019;11:301-305.

11. Losciale JM, Zdeb RM, Ledbetter L, Reiman MP, Sell TC. The association between passing return-to-sport criteria and second anterior cruciate ligament injury risk: A systematic review with meta-analysis. J Orthop Sports Phys Ther 2018:49:43-54.

12. Webster KE, Hewett TE. What is the evidence for and validity of return-to-sport testing after anterior cruciate ligament reconstruction surgery? A systematic review and meta-analysis. Sports Med 2019;49:917-929.

13. Xerogeanes JW. Quadriceps tendon graft for anterior cruciate ligament reconstruction: The graft of the future. Arthroscopy 2019;35:696-697.

14. Pennock AT, Johnson KP, Turk RD, et al. Transphyseal anterior cruciate ligament reconstruction in the skeletally immature: Quadriceps tendon autograft versus hamstring tendon autograft. Orthop J Sports Med 2019;7: 2325967119872450

15. Saper MG. Quadriceps tendon autograft anterior cruciate ligament reconstruction with independent suture tape reinforcement. Arthrosc Tech 2018;7:e1221-e1229.

16. Hoggan Scientific website. Available at: https:// hogganscientific.com/product/microfet2-muscle-testerdigital-handheld-dynamometer/. Accessed December 24, 2020

17. Kocher MS, Smith JT, Iversen MD, et al. Reliability, validity, and responsiveness of a modified International Knee Documentation Committee Subjective Knee Form (PediIKDC) in children with knee disorders. Am J Sports Med 2011;39:933-939.

18. Tegner Y, Lysholm J. Rating systems in the evaluation of knee ligament injuries. Clin Orthop 1985;198:43-49.

19. Martin-Alguacil JL, Arroyo-Morales M, Martin-Gomez JL, et al. Strength recovery after anterior cruciate ligament reconstruction with quadriceps tendon versus hamstring tendon autografts in soccer players: A randomized controlled trial. Knee 2018;25:704-714.

20. Fischer F, Fink C, Herbst E, et al. Higher hamstring-toquadriceps isokinetic strength ratio during the first post-operative months in patients with quadriceps tendon compared to hamstring tendon graft following ACL reconstruction. Knee Surg Sports Traumatol Arthrosc 2018;26:418-425.

21. Lee JK, Lee S, Lee MC. Outcomes of anatomic anterior cruciate ligament reconstruction: Bone-quadriceps tendon graft versus double-bundle hamstring tendon graft. Am J Sports Med 2016;44:2323-2329.

22. Hunnicutt JL, Gregory CM, McLeod MM, Woolf SK, Chapin RW, Slone HS. Quadriceps recovery after anterior cruciate ligament reconstruction with quadriceps tendon versus patellar tendon autografts. Orthop J Sports Med 2019;7:2325967119839786.

23. Letchford R, Button K, Sparkes V, van Deursen RWM. Assessing activity participation in the ACL injured population: A systematic review of activity rating scale measurement properties. Phys Ther Rev 2012;17:99-109.

24. Nwachukwu BU, Chang B, Voleti PB, et al. Preoperative short form health survey score is predictive of return to play and minimal clinically important difference at a minimum 2-year follow-up after anterior cruciate ligament reconstruction. Am J Sports Med 2017;45:2784-2790.

25. Ardern CL, Taylor NF, Feller JA, Whitehead TS, Webster KE. Sports participation 2 years after anterior cruciate ligament reconstruction in athletes who had not returned to sport at 1 year: A prospective follow-up of physical function and psychological factors in 122 athletes. Am J Sports Med 2015;43:848-856.

26. Welling W, Benjaminse A, Seil R, Lemmink $\mathrm{K}$, Zaffagnini S, Gokeler A. Low rates of patients meeting return to sport criteria 9 months after anterior cruciate ligament reconstruction: A prospective longitudinal study. Knee Surg Sports Traumatol Arthrosc 2018;26: 3636-3644.

27. Smith CA, Chimera NJ, Warren M. Association of y balance test reach asymmetry and injury in division I athletes. Med Sci Sports Exerc 2015;47:136-141.

28. Losciale JM, Bullock G, Cromwell C, Ledbetter L, Pietrosimone L, Sell TC. Hop testing lacks strong association with key outcome variables after primary anterior cruciate ligament reconstruction: A systematic review. Am J Sports Med 2020;48:51 1-522.

29. Davies WT, Myer GD, Read PJ. Is it time we better understood the tests we are using for return to sport decision making following ACL reconstruction? a critical review of the hop tests. Sports Med 2020;50:485-495. 